\title{
Sex-specific pathways in early cardiac response to pressure overload in mice
}

\author{
Henning Witt • Carola Schubert • Juliane Jaekel • Daniela Fliegner • Adam Penkalla • Klaus Tiemann • \\ Joerg Stypmann • Stefan Roepcke • Sebastian Brokat - Shokoufeh Mahmoodzadeh • Eva Brozova • \\ Mercy M. Davidson • Patricia Ruiz Noppinger • Christian Grohé • Vera Regitz-Zagrosek
}

Received: 5 February 2008 /Revised: 30 May 2008 / Accepted: 18 June 2008 / Published online: 30 July 2008

(C) Springer-Verlag 2008

\begin{abstract}
Pressure overload (PO) first causes cardiac hypertrophy and then heart failure (HF), which are associated with sex differences in cardiac morphology and function. We aimed to identify genes that may cause HF-related sex differences. We used a transverse aortic constriction (TAC) mouse model leading to hypertrophy without sex differences in cardiac function after 2 weeks, but with sex differences in hypertrophy 6 and 9 weeks after TAC. Cardiac gene expression was analyzed 2 weeks after surgery. Deregulated genes were classified into functional gene ontology (GO) categories and used for pathway analysis. Classical marker genes of hypertrophy were similarly upregulated in both sexes $(\alpha$-actin, ANP, BNP, CTGF). Thirty-five genes controlling mitochondrial function (PGC-1, cytochrome oxidase, carnitine palmitoyl transferase, acyl-CoA dehydrogenase, pyruvate dehydrogenase kinase) had lower expression in males compared to
\end{abstract}

Henning Witt and Carola Schubert contributed equally to this work.

Electronic supplementary material The online version of this article (doi:10.1007/s00109-008-0385-4) contains supplementary material, which is available to authorized users.

H. Witt $\cdot$ C. Schubert $\cdot$ J. Jaekel $\cdot$ D. Fliegner $\cdot$ S. Brokat $\cdot$

S. Mahmoodzadeh $\cdot$ E. Brozova $\cdot$ P. Ruiz Noppinger $\cdot$

V. Regitz-Zagrosek

Berlin Institute of Gender in Medicine (GiM),

Charité-Universitaetsmedizin Berlin,

Luisenstraße 65, 10117 Berlin, Germany

H. Witt • C. Schubert · J. Jaekel • D. Fliegner · A. Penkalla •

S. Brokat $\cdot$ S. Mahmoodzadeh - E. Brozova - P. Ruiz Noppinger

V. Regitz-Zagrosek $(\triangle)$

Center for Cardiovascular Research (CCR),

Charité-Universitaetsmedizin Berlin, Hessische Str. 3-4,

10115 Berlin, Germany

e-mail: vera.regitz-zagrosek@charite.de females after TAC. Genes encoding ribosomal proteins and genes associated with extracellular matrix remodeling exhibited relative higher expression in males (collagen 3, matrix metalloproteinase 2, TIMP2, and TGF $\beta 2$, all about twofold) after TAC. We confirmed $87 \%$ of the gene expression by real-time polymerase chain reaction. By GO classification, female-specific genes were related to mitochondria and metabolism and males to matrix and biosynthesis. Promoter studies confirmed the upregulation of PGC-1 by E2. Less downregulation of metabolic genes in female hearts and increased protein synthesis capacity and deregulation of matrix remodeling in male hearts characterize the sex-specific early response to PO. These differences could contribute to subsequent sex differences in cardiac function and HF.

Keywords Pressure overload · Hypertrophy . Gene expression $\cdot$ Sex $\cdot$ Heart

K. Tiemann · J. Stypmann • C. Grohé

Medizinische Klinik und Polyklinik II, Universitaetsklinikum Bonn, Sigmund-Freud-Str. 25, 53105 Bonn, Germany

H. Witt $\cdot$ S. Roepcke $\cdot$ P. Ruiz Noppinger

Max-Planck-Institute for Molecular Genetics Berlin,

Ihnestr. 63-73,

14195 Berlin, Germany

V. Regitz-Zagrosek

German Heart Institute Berlin (DHZB),

Augustenburger Platz 1, 13353 Berlin, Germany 


\section{Introduction}

Pressure overload (PO), as in hypertension or aortic stenosis, initially leads to left ventricular hypertrophy (LVH) and later to heart failure (HF). In the population, women with aortic stenosis develop less HF at similar mechanical load than men. HF affects about $10 \%$ of persons aged 70 years and increases thereafter [1-4]. Women more commonly develop $\mathrm{HF}$ with preserved ejection fraction, whereas men more often have HF with reduced ejection fraction [5-7]. In addition, women with HF have a better outcome at similarly reduced systolic function [8,9]. Estrogens improve myocardial adaptation to PO in hypertensive women $[10,11]$ and in mouse models [12, 13]. Several transgenic LVH models have shown that female animals develop HF and death at a later time point than males [14-16]. PO in rodent models leads to LVH with normal systolic function at an early stage and impaired systolic function at later stages with significant sex differences [17-19]. The exact time point when sex differences appear depends on the model, the degree of $\mathrm{PO}$, animal age, and strain. In a rat transverse aortic constriction (TAC) model with moderate PO, males showed more LVH and depressed contractile reserve than females [18, 20]. With severe PO, male mice had worse LVH than females by 2 weeks [19]. Gene expression has identified hypertrophyrelated genes mainly in males or in mixed populations [17, 21-23]. The profiles suggested pathways that included functionally related pro- and antihypertrophy genes or estrogen receptor $(E R \alpha$ and $E R \beta)$-driven genes [22, 24]. However, only a single microarray study on sex differences in cardiac hypertrophy has been published so far in mice [17]. Sex differences that might underlie the functional differences at later stages were not identified. We aimed to study this issue and used a novel bioinformatics approach to define networks of functionally related genes $[25,26]$.

\section{Materials and methods}

\section{Animal experiments}

All animal experiments followed the "Principles of laboratory animal care" (NIH publication No. 86-23, revised 1985) as well as the current version of the German Law on the Protection of Animals.

In the first cohort, we randomized 12-14-week-old male and female $\mathrm{C} 57 \mathrm{BL} / 6 \mathrm{~J}$ mice to either sham operation or banding of the thoracic aorta [13]. Four animals per group (shamoperated males, females, TAC males, and females) underwent

M. M. Davidson

College of Physicians and Surgeons, Columbia University, New

York, NY, USA echocardiography 2 weeks after surgery and were killed 2 days later. A two-dimensional short-axis view and M-mode tracings of the left ventricle were obtained with a $45-\mathrm{MHz}$ transducer (Vevo $770^{\mathrm{TM}}$, VisualSonics, Canada). Wall thickness and left ventricular diameters (LVEDD/LVESD) were measured according to the American Society for Echocardiography. Left ventricles were immediately frozen in liquid nitrogen and used for microarray analysis and real-time polymerase chain reaction (PCR). Hemodynamic parameters were evaluated in a second cohort of $4 \times 4$ male and female mice that underwent TAC or sham surgery, parallel to the main experiment. A pressure transducer catheter (Millar Instruments) was introduced into the right carotid artery and advanced into the left ventricle to assess ventricular function. Data were analyzed using a computerized data acquisition system (Power Lab, ADI Instruments, Melbourne, Australia) [13]. In a third cohort, echocardiography was done at weeks 2,6 , and 9 after TAC or sham surgery to determine LV mass ( $n=62$, males/females/sham/TAC).

\section{Microarray analysis}

Four individual hearts per group from cohort 1 (sham male, sham female, TAC male, TAC female) were analyzed on 16 single arrays. The amplification and labeling of the RNA samples were carried out according to standard protocols (Affymetrix, Santa Clara, CA, USA). Fifteen micrograms of each cRNA sample was hybridized to Affymetrix RAE 430A GeneChip arrays. Arrays were scanned at $3 \mathrm{~mm}$ resolution using the Affymetrix GeneChip System confocal scanner 2500 (Electronic supplementary material), and raw signal intensities were normalized globally with a scaling factor using Affymetrix GeneChip ${ }^{\circledR}$ Operating Software (GCOS). The gene expression data were deposited in the Gene Expression Omnibus (GEO) database (http://www.ncbi.nlm. nih.gov/projects/geo/) as GEO accession GSE6970.

\section{Quantification of mRNA by real-time PCR}

Total RNA preparation (RNABee, BIOZOL, Germany), desoxyribonuclease (DNase) digestion (DNAse I, Invitrogen, Germany), and reverse transcription (SuperScript, Invitrogen, Germany) were performed according to the manufacturer's recommendations. A "hot start" real-time PCR procedure with SYBR Green was performed in duplicates with the ABI Prism 7700 Sequence Detection System. The mRNA content of the target genes was normalized to the expression of GAPDH in the same sample. Primers for all target genes were designed using Primer3 Software (Table e1, Electronic supplementary material) [27]. To correct differences in PCR efficiencies, a calibration curve containing $25,12.5,6.25,3.13$, and $1.56 \mathrm{ng}$ cDNA template pooled from all samples analyzed was run with each primer pair. 
Statistical analysis

Morphological and functional data were analyzed using the Mann-Whitney test and one-way analysis of variance (ANOVA) followed by Tukey's test. Differences were considered significant at $p<0.05$. For the identification of differentially expressed genes in the comparison of two conditions with microarrays (male vs. female, TAC vs. sham), we used the significance analysis of microarrays (SAM) [28]. The SAM statistic identifies significant changes in gene expression by performing a set of genespecific $t$ tests. For each gene, a score is calculated on the basis of expression change relative to the standard deviation of repeated measurements for that gene. Genes with scores greater than a threshold delta were defined as significantly deregulated. Manual adjustment of this threshold delta allows the identification of smaller or larger gene cohorts. In addition, based on random permutations of all measurements, a false discovery rate was estimated. For the identification of differentially expressed genes in the comparison of four conditions (sham female, sham male, TAC female, and TAC male) we used a two-way ANOVA to test for the interaction between hypertrophy (TAC/sham) and sex (male/female) without correction for multiple testing. A $p$ value $<0.05$ indicates that the gene is deregulated differentially after PO in males and females. For functional annotation and pathway analysis, we classified the genes as relatively upregulated in females or males, respectively. For example, a relative upregulation in females would correspond to: first, a relative stronger induction (female TAC/female sham $>$ male TAC/male sham $>1)$; second, a weaker repression $(1>$ female $\mathrm{TAC} /$ female sham $>$ male TAC/male sham); or third, an opposite regulation (female TAC/female sham $>1>$ male TAC/male sham) of gene expression.

Functional annotation and pathway analysis

For the detection of gene ontology (GO, www.geneontology.org) categories and Kyoto Encyclopedia of Genes and Genomes (KEGG, www.kegg.com) pathways with a significant overrepresentation of genes in a given group compared to the whole genome, the web-based Database for Annotation Visualization and Integrated Discovery tool (DAVID, National Institute of Allergy and Infectious Disease) was used $[25,26]$. Fisher's exact test was applied to determine whether or not the proportion of those genes falling into each GO category or KEGG pathway differed significantly between the input data set and the whole genome. Networks of biologically related genes were created with the help of the Ingenuity Pathways Analysis (Ingenuity ${ }^{\circledR}$ Systems, www.ingenuity.com). The sex-specific regulated genes obtained from the microarray analysis were used to build literature-based sex-specific networks. In the resulting networks, genes or gene products are represented as nodes, and the biological relationship between two nodes is shown as a line. All lines are supported by at least one reference from literature, from a textbook, or from canonical information stored in the Ingenuity Pathways Knowledge Base. For each network, a score is calculated based on the $p$ value of a right-tailed Fisher's exact test. This score calculates the approximate fit between each network and the focus genes from the input data set and indicates whether or not a network contains more genes than expected by chance.

Cell culture and transient transfection reporter assays

Human genomic DNA isolated from peripheral blood samples of healthy volunteers was used as template to generate the reporter construct containing the $5^{\prime}$-flanking region of the PGC- $1 \alpha$ gene by PCR with PGC- $1 \alpha-1.7 \mathrm{~kb}-$ Fw: GATCGGATCCGTGCTGGTGAACTGTATTCAGC, PGC- $1 \alpha-0.6$ kb-Fw: GATCGGATCCCGCTTTCAAACACTCCCTCAATG, respectively, and PGC- $1 \alpha-\mathrm{RV}$ : GATCGGATCCTCCTGAATGACGCCAGTCAAGC primers. PGC- $1 \alpha$ promoter fragments $(-1,655 \mathrm{bp} /+114 \mathrm{bp}$ and $-515 \mathrm{bp} /+114 \mathrm{bp}$, relative to the transcriptional start site +1 ) were cloned into pJET1.2 vector (Fermentas) and confirmed by sequencing. The individual promoter fragments were subcloned into the luciferase reporter vector pGL3-basic (Promega) using BglII restriction sites in sense and antisense orientation. AC16 cells (human cardiomyocyte cell line, Davidson et al. [29]) were cultured in phenol red-free DMEM/F12, supplemented with $12.5 \%$ charcoal-stripped fetal calf serum (FCS; Biochrom AG), penicillin/streptomycin $(100 \mathrm{U} / \mathrm{ml} /$ $100 \mu \mathrm{g} / \mathrm{ml}$, PAA), and amphotericin B $(0.25 \mu \mathrm{g} / \mathrm{ml})$ at $37^{\circ} \mathrm{C}$ under $5 \% \mathrm{CO}_{2}$. For transient transfection experiments, $500 \mathrm{ng}$ of the PGC- $1 \alpha$ promoter-luciferase reporter construct and $100 \mathrm{ng}$ of the internal reference Renilla luciferase reporter plasmid phRL-TK vector (Promega) were co-transfected into AC16 cells using FuGENE ${ }^{\circledR}$ HD reagent (Roche Diagnostics). In some experiments, ER $\alpha$ pSG5 plasmid (HEGO-vector) or its empty vector pSG5 were co-transfected by using a similar transfection procedure. Twenty-four hours after transfection, cells were treated with or without E2 $\left(10^{-8} \mathrm{M}\right.$, Sigma $)$ in phenol redfree DMEM containing 2.5\% charcoal-stripped FCS for additional $24 \mathrm{~h}$. Firefly and Renilla luciferase activities were measured using the Dual-Glo ${ }^{\mathrm{TM}}$ Luciferase Assay System (Promega). Transfections were carried out in triplicates for at least three times. The authors had full access to the data and take responsibility for its integrity. All authors have read and agreed to the manuscript as written. 
Fig. 1 Morphology and hemodynamic measurements. Left ventricular weight to tibia length (LV/TL; a) ratio, posterior wall thickness (b), left ventricular systolic pressure (LVSP; $\mathbf{c}$ ), and maximum and minimum rate of rise of ventricular pressure $(+\mathrm{dp} / \mathrm{dtmax}$ and $-\mathrm{dp} / \mathrm{dtmin}(1 \mathrm{~d}))$ are shown. In a mean of sham male (sham-m) mice was set as $100 \%$ and sham female (sham-f) as well as TAC mice of both sexes $(T A C-m, T A C-f)$ are expressed as percentage of Sham$\mathrm{m}$. Data are presented as mean \pm SEM. $* p<0.05 ; * * p<0.001$ (Mann-Whitney test); sham-m are shown in white bars, TAC-m in white bars with black pattern, sham-f are shown in gray bars, and TAC-f in gray bars with black pattern a

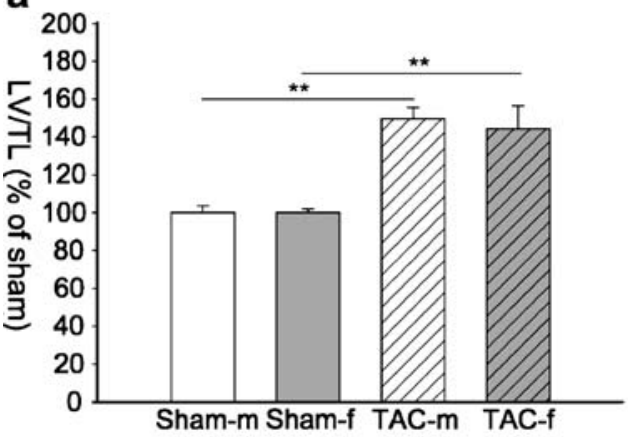

C

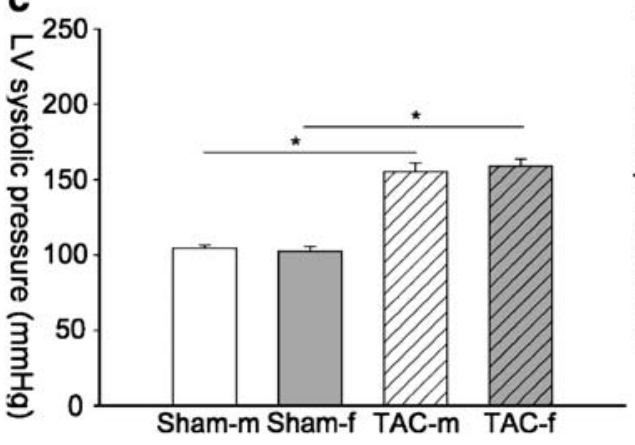

b
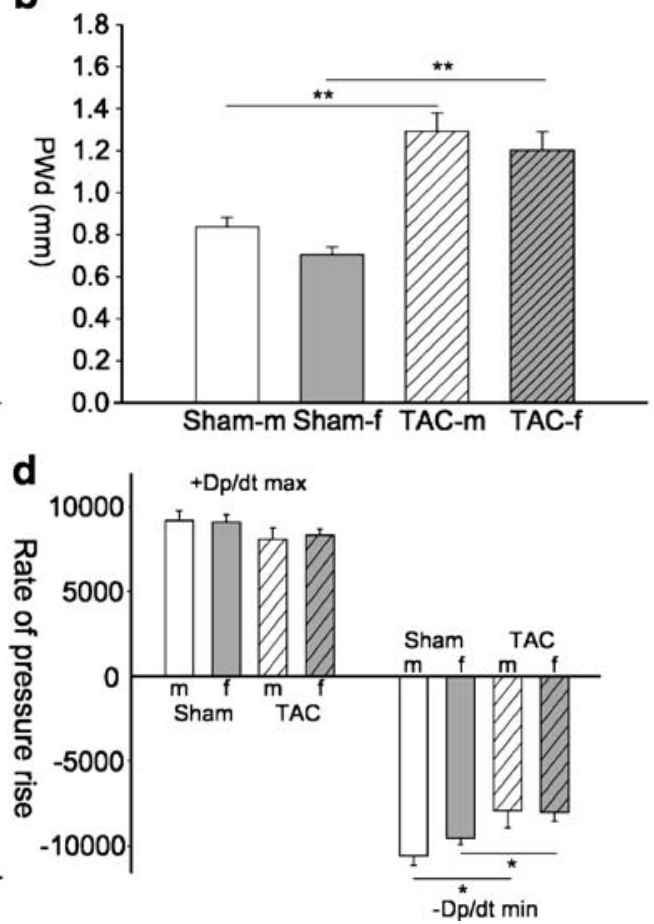

\section{Results}

Sex differences in LVH were present at 6 weeks but not at 2 weeks

Two weeks after TAC, left ventricular weight to tibia length (LV/TL) was similarly increased in male and female TAC animals (males $150 \pm 5 \%$; females $144 \pm 10 \%$; ns; Fig. 1a). Echocardiography confirmed that TAC induced a similar increase in wall thickness in females and males (Fig. 1b). Heart rate (data not shown), LV pressure, dp/dtmax, and dp/ dtmin were similar in both sexes (Fig. 1c,d). In contrast, significant sex differences in LV mass were present at week 6 and 9 after TAC (Fig. 2).
PO-induced gene regulation

We analyzed the effect of TAC on classical hypertrophy markers in independent comparisons separately in the two sexes (TAC female vs. sham female or TAC male vs. sham male). Eight hundred thirty genes (258 upregulated and 572 downregulated) were responsive to pressure overload in females and 965 genes (369 upregulated and 596 downregulated) in males (false discovery rate $<1 \%$, SAM; Fig. 3 ). Typical markers of hypertrophy such as alpha skeletal actin, natriuretic peptide precursor A, natriuretic peptide precursor $\mathrm{B}$, procollagen type I, and connective tissue growth factor were induced significantly in both sexes to a similar degree (Fig. 4a).
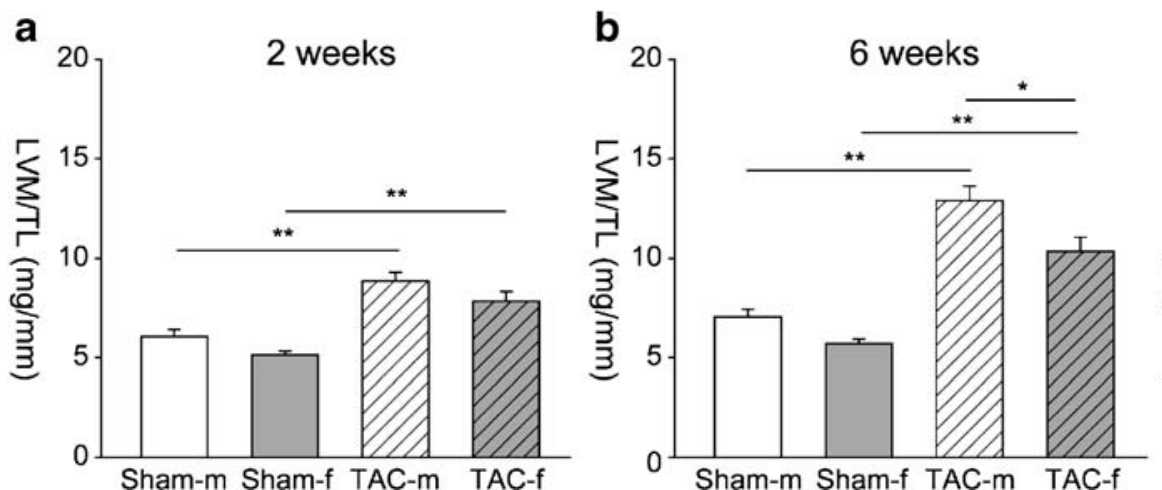

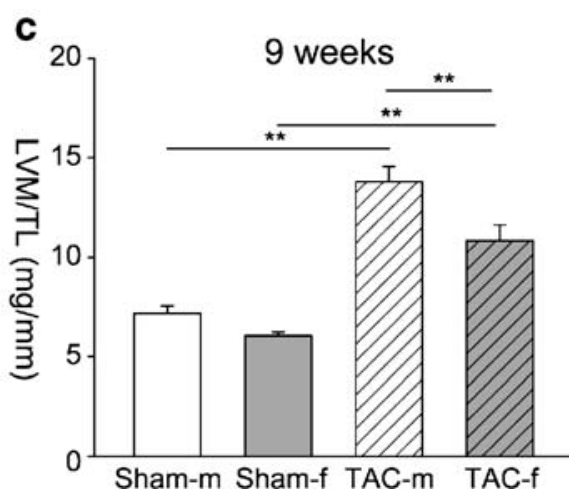

$*^{*} p<0.001$ (one-way ANOVA followed by Tukey's test); Sham-m are shown in white bars, TAC-m in white bars with black pattern, sham-f are shown in gray bars, and TAC-f in gray bars with black pattern
Fig. 2 Sex differences at later stages of cardiac hypertrophy ventricular mass (calculated form echocardiography) to tibia length $(\mathrm{LVM} / \mathrm{TL})$ ratios 2 weeks (a), 6 weeks (b), and 9 weeks (c) after surgery in cohort 3 are shown. Data are presented as mean \pm SEM. ${ }^{*} p<0.05$; 
Fig. 3 Design and statistical analysis of the microarray experiments. Four individual samples for each condition were hybridized onto Affymetrix RAE 430A GeneChip arrays. The statistical significance of the influence of either sex or hypertrophy (one factor) was calculated using the significance analysis of microarrays (SAM). To calculate sex- and hypertrophy-specific gene expression (two factors) ANOVA was used. The set of deregulated genes $(n=653)$ was split in a female- and a malespecific subset which were further analyzed with DAVID and Ingenuity Pathways Analysis

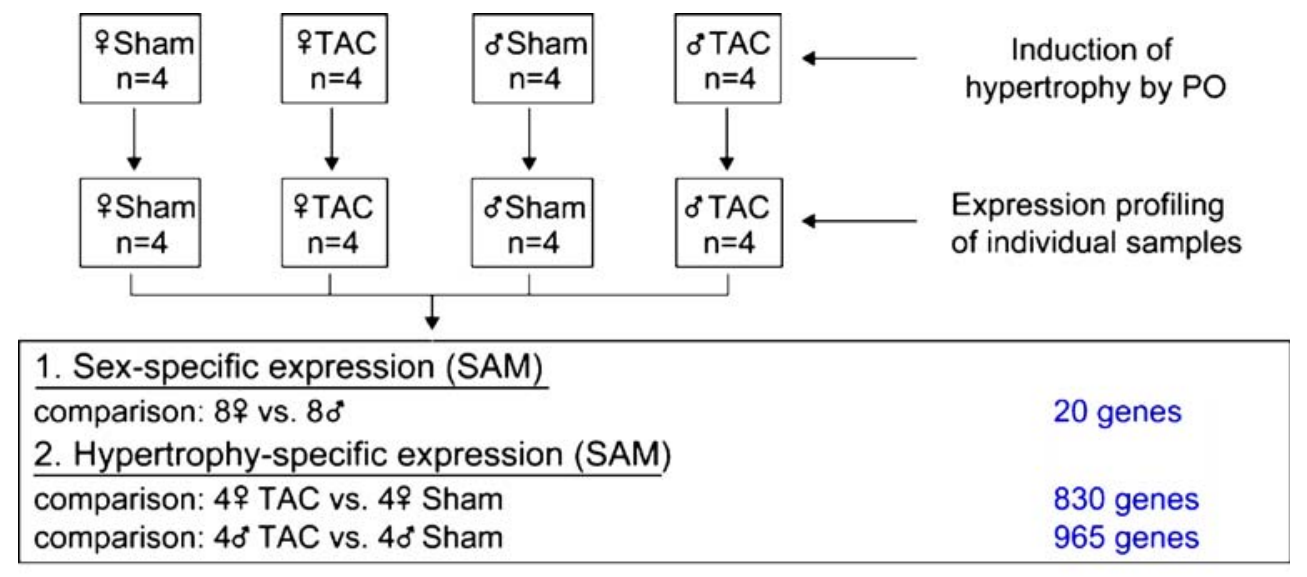

\begin{tabular}{|c|c|c|c|}
\hline \multicolumn{4}{|c|}{ 3. Sex- and hypertrophy-specific expression (2-factor ANOVA) } \\
\hline \multicolumn{3}{|c|}{$\begin{array}{l}\text { comparison: } 49 \text { Sham, } 49 \text { TAC, } 40^{\circ} \text { Sham, } 4 \sigma^{\circ} \mathrm{TAC} \\
\text { Subset a: relative upregulation in females: }\end{array}$} & 653 genes $\neg$ \\
\hline \multicolumn{3}{|c|}{ \&TAC / \&Sham > ठTAC / ठSham } & 338 genes 4 \\
\hline \multicolumn{4}{|l|}{ Subset b: relative upregulation in males: } \\
\hline \multicolumn{3}{|l|}{ oTAC / óSham > \&TAC / \&Sham } & 315 genes 4 \\
\hline 4. DAVID & & 5. Ingenuity & \\
\hline $\begin{array}{l}\text { Identification of enriched gene ontology } \\
\text { categories in subset a or b }\end{array}$ & & $\begin{array}{l}\text { Identification of net } \\
\text { related genes in su }\end{array}$ & $\begin{array}{l}\text { rks of functionally } \\
\text { et } a \text { or } b\end{array}$ \\
\hline
\end{tabular}

Sex-specific gene expression

A small group of genes showed differential expression between males and females (Fig. 4b, c) independent from TAC (all males vs. all females). As in previous studies for somatic tissues [30], the overall number was relatively low
(20 genes, false discovery rate $<10 \%$, SAM). Six genes showed higher expression in females including several Xlinked genes. Fourteen genes showed higher expression in males including members of the $\mathrm{X}$-degenerated region of the $\mathrm{Y}$ chromosome (Table e2, Electronic supplementary material).

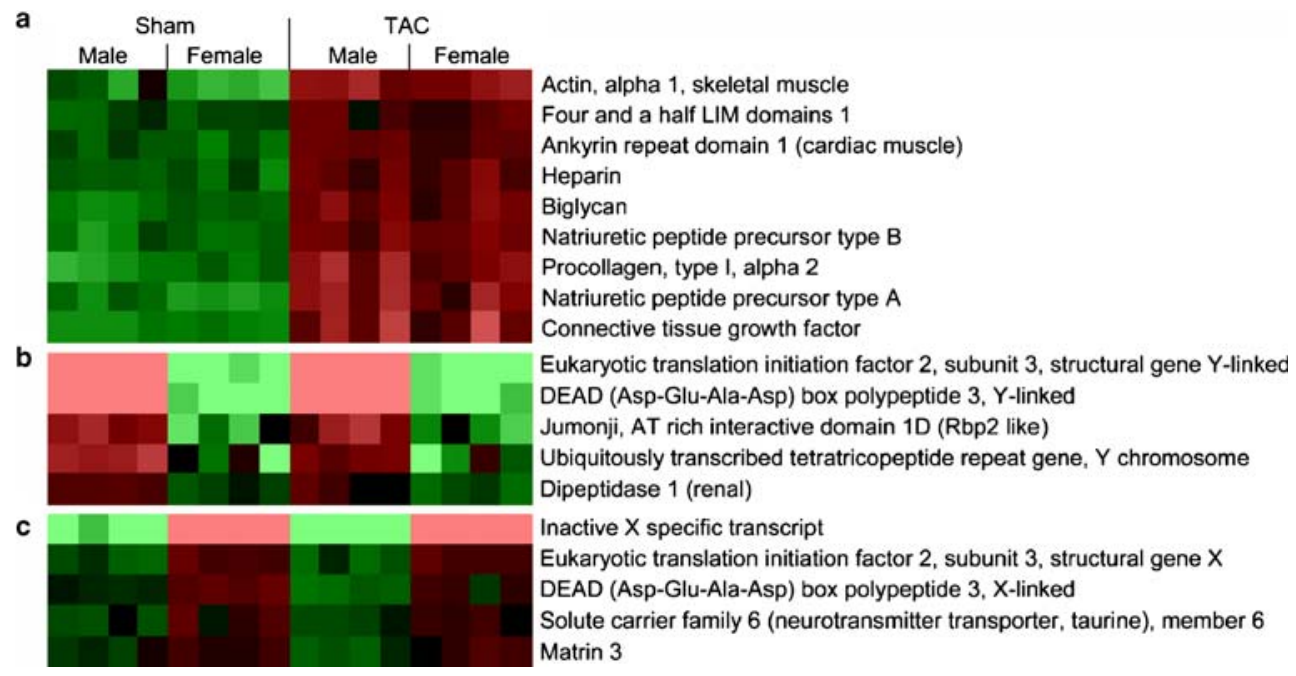

Fig. 4 Hypertrophy-induced and sex-specific gene expression. mRNA expression levels of a selection of hypertrophy markers (a) and a selection of Y-linked (b) and X-linked (c) genes analyzed by microarray are shown. The mean mRNA expression level of the 16 individual samples is set as zero and shown in black for each gene. A relative upregulation to that mean value is shown in red. The brighter the red appears the stronger the mRNA expression is upregulated. A relative downregulation to the mean value is shown in green. The brighter the green appears the stronger the mRNA expression is downregulated. False discovery rate (TAC vs. sham) $<1 \%$ (SAM) false discovery rate (female vs. male) $<10 \%$ (SAM) 
Sex-specific gene regulation in hypertrophy by microarray and confirmatory real-time PCR

TAC-induced PO led to the differential regulation of 653 genes in females and males $(p<0.05$, ANOVA statistics; Table e3, Electronic supplementary material). Three hundred thirtyeight genes showed a relative upregulation in females, which could be due to a relative stronger induction or to a weaker repression in females or an opposite regulation in both sexes. These genes are designated female specific. Vice versa, 315 genes showed a relative upregulation in males and are designated male specific. Forty-five genes that differed significantly in their regulation between both sexes after 2 weeks of $\mathrm{TAC}$ in the microarray analysis $(p<0.05$, ANOVA) were selected for confirmation by real-time PCR (Table e4, Electronic supplementary material). To compare the measurements of the four conditions (sham female, sham male, TAC female, and TAC male) between the two techniques the calculated value (female TAC/sham) divided by (male TAC/ sham) was plotted for both approaches (Fig. 5). Relative upor downregulation was confirmed in $87 \%$ of these genes. For all genes measured, the relationship of TAC-dependent gene expression in the two sexes correlated well between the microarray analysis and the real-time PCR $(p<0.001, r=0.813)$.

Functional classification of sex-specific regulated genes

The following data analysis steps are based on the two sets of sex-specific regulated genes: 338 female-specific genes and 315 male-specific genes. These genes were classified according to their gene ontology category and presence in KEGG

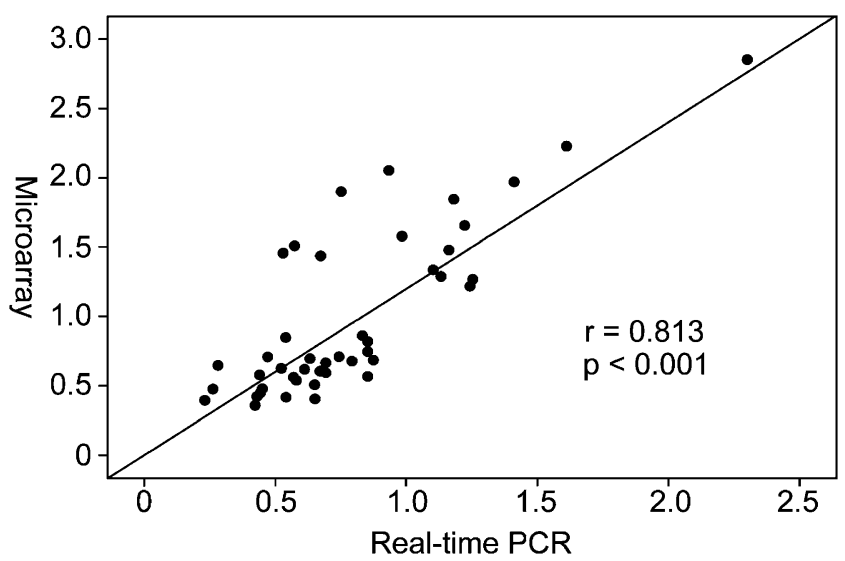

Fig. 5 Correlation of the ratios from the microarray and real-time PCR data set. Genes that differed significantly $(p<0.05)$ in their regulation by TAC between both sexes in the microarray analysis were selected based on their biological relevance and were validated with the same samples by real-time PCR analysis. The ratio (TAC-f/sham-f divided by TAC-m/sham-m) calculated from the microarray data set correlated well with the ratio calculated from the real-time PCR data $(r=0.806, p<0.001)$. A complete list of these genes is shown in Table e4 (Electronic supplementary material) pathways with the web-accessible DAVID program. The female-specific genes were significantly overrepresented in the "biological process" categories of macromolecule metabolism, cellular metabolism, and primary metabolism and in the "cellular component" categories related to mitochondria (Table e5). The mainly affected KEGG pathways were insulin signaling and propanoate and pyruvate metabolism (Table 1, Table e6, complete list as Electronic supplementary material). In contrast, male-specific genes were overrepresented in the "biological process" categories of biosynthesis, cell organization and biogenesis, and macromolecule metabolism (Table 1). Within the category biosynthesis, particularly a group of 20 ribosomal genes were all relatively stronger and significantly upregulated in males (Table 2). Accordingly, male-specific genes were overrepresented in the "cellular component" category and KEGG pathway ribosome (Table e6, Electronic supplementary material).

For the identification of functionally related transcripts, the two sets of genes with relative overexpression in females or males were used to build networks with the Ingenuity Pathways Analysis software. The most prominent female network included 29 genes of the import data set and six added external genes (Fig. 6a, Table e7, Electronic supplementary material). A significant number of these genes were related to mitochondrial energy metabolism.

The most prominent male network included 32 genes and three external genes (Fig. 6b, Table e7, Electronic supplementary material). In the male-specific network, genes related to extracellular matrix synthesis were overrepresented. These genes included procollagen type III alpha 1 (Col3a1) and procollagen type $\mathrm{V}$ alpha 2 (Col5a2), matrix metalloproteinase 2 (MMP2), tissue inhibitor of metalloproteinase 4 (TIMP4), transcription factors from the sons of mothers against decapentaplegic (SMAD) family, and transforming growth factor beta2 (TGF 32 ). The second best male network included the prohypertrophic transcription factor myocyte enhancer factor 2C (MEF2; Fig. e1, Table e7, Electronic supplementary material). MEF2c was downregulated in females, whereas the gene was almost unchanged in males (ratio female/male Array $=$ 0.54 ; ratio female $/$ male $_{\mathrm{RT}-\mathrm{PCR}}=0.58$ ).

Regulation of the PGC- $1 \alpha$ promoter activity by E2

To determine the effect of estrogen signaling on the activity of the PGC- $1 \alpha$ promoter, we co-transfected PGC- $1 \alpha$ expression constructs with or without the $\mathrm{ER} \alpha$ plasmid into $\mathrm{AC} 16$ cells. After $24 \mathrm{~h}$ of E2 treatment, the relative luciferase activities of PGC- $1 \alpha$ reporter constructs did not change significantly in response to $\mathrm{E} 2$ alone in $\mathrm{AC} 16$ cells, whereas in the presence of $\mathrm{ER} \alpha$ plasmid, the transcriptional activation of all PGC- $1 \alpha$ reporter constructs were significantly elevated in response to E2 (Fig. 7). As negative control, the reporter construct, which contained PGC- $1 \propto 1.7 \mathrm{~kb}$ promoter sequence in antisense 
Table 1 Genes with sex differences in their expression pattern were GO-annotated at level 4 of the categories "biological process" and "cellular localization" and were sorted in KEGG pathways

\begin{tabular}{|c|c|c|c|}
\hline & Category/pathway & No. of genes & $p$ value \\
\hline \multicolumn{4}{|c|}{ Ratio $>1$ (relatively upregulated in females) } \\
\hline \multirow[t]{3}{*}{ Biological process } & Protein metabolism & 81 & 0.00002348 \\
\hline & Cellular macromolecule metabolism & 78 & 0.00002692 \\
\hline & Biopolymer metabolism & 70 & 0.00005013 \\
\hline \multirow[t]{3}{*}{ Cellular component } & Mitochondrion & 35 & 0.00000492 \\
\hline & Intracellular membrane-bound organelle & 131 & 0.00001389 \\
\hline & Mitochondrial inner membrane & 15 & 0.00016492 \\
\hline \multirow[t]{3}{*}{ KEGG pathway } & Propanoate metabolism (MMU00640) & 4 & 0.03988610 \\
\hline & Pyruvate metabolism (MMU00620) & 4 & 0.05525333 \\
\hline & Insulin signaling (MMU04910) & 7 & 0.06556266 \\
\hline \multicolumn{4}{|c|}{ Ratio $<1$ (relatively upregulated in males) } \\
\hline \multirow[t]{3}{*}{ Biological process } & Macromolecule biosynthesis & 38 & $<0.00000001$ \\
\hline & Protein metabolism & 88 & 0.00000002 \\
\hline & Cellular biosynthesis & 44 & 0.00000002 \\
\hline \multirow[t]{3}{*}{ Cellular component } & Ribosome & 19 & 0.00000003 \\
\hline & Cytosolic ribosome (sensu Eukaryota) & 9 & 0.00000257 \\
\hline & Intracellular membrane-bound organelle & 140 & 0.00002176 \\
\hline \multirow[t]{3}{*}{ KEGG pathway } & Ribosome (MMU03010) & 16 & 0.00000001 \\
\hline & Ribosome (HSA03010) & 6 & 0.00026498 \\
\hline & Focal adhesion (MMU04510) & 12 & 0.01538544 \\
\hline
\end{tabular}

Categories and pathways showing the highest significance in the overrepresentation of genes are shown. Female-specific genes are significantly overrepresented in the different categories of metabolism whereas male-specific genes are overrepresented in the categories of biosynthesis.

Table 2 Ribosomal genes with relative upregulation in males

\begin{tabular}{|c|c|c|c|c|c|}
\hline Probe set ID $^{\mathrm{a}}$ & Gene title & Gene symbol & Ratio female vs. male & Ratio male vs. female & $p$ value \\
\hline 1418112_at & Mitochondrial ribosomal protein L10 & Mrpl10 & 0.68 & 1.47 & 0.050 \\
\hline 1415690_at & Mitochondrial ribosomal protein L27 & Mrpl27 & 0.78 & 1.27 & 0.015 \\
\hline 1424372_at & Mitochondrial ribosomal protein L32 & Mrpl32 & 0.77 & 1.29 & 0.040 \\
\hline 1416984_at & Mitochondrial ribosomal protein S18A & Mrps18a & 0.72 & 1.38 & 0.002 \\
\hline 1415942_at & Ribosomal protein L10 & Rpl10 & 0.80 & 1.24 & 0.014 \\
\hline 1455485_x_at & Ribosomal protein L13a & Rpl13a & 0.80 & 1.25 & 0.020 \\
\hline 1437005_a_at & Ribosomal protein L18 & Rp118 & 0.78 & 1.27 & 0.003 \\
\hline 1455767_x_at & Ribosomal protein L21 & Rpl21 & 0.70 & 1.42 & 0.005 \\
\hline 1415701_x_at & Ribosomal protein L23 & Rpl23 & 0.80 & 1.25 & 0.023 \\
\hline 1437975_a_at & Ribosomal protein L23a & Rpl23a & 0.83 & 1.21 & 0.050 \\
\hline 1448846_a_at & Ribosomal protein L29 & Rpl29 & 0.82 & 1.22 & 0.029 \\
\hline 1424000_a_at & ribosomal protein $\mathrm{S} 11$ & Rps11 & 0.81 & 1.23 & 0.010 \\
\hline 1416088_a_at & Ribosomal protein $\mathrm{S} 15$ & Rps 15 & 0.83 & 1.21 & 0.014 \\
\hline 1416404_s_at & Ribosomal protein S16 & Rps16 & 0.75 & 1.34 & 0.027 \\
\hline 1428530_x_at & Ribosomal protein S24 & Rps24 & 0.76 & 1.32 & 0.030 \\
\hline 1422475_a_at & Ribosomal protein $\mathrm{S} 3 \mathrm{a}$ & Rps3a & 0.86 & 1.17 & 0.029 \\
\hline 1416054_at & Ribosomal protein S5 & Rps5 & 0.76 & 1.32 & 0.049 \\
\hline 1455364_a_at & Ribosomal protein S7 & Rps7 & 0.71 & 1.41 & 0.006 \\
\hline 1455319_x_at & Ribosomal protein S8 & Rps8 & 0.82 & 1.21 & 0.031 \\
\hline 1449221_a_at & Ribosome binding protein 1 & Rrbp1 & 0.42 & 2.40 & 0.016 \\
\hline
\end{tabular}

$p$ value indicates interaction of sex and hypertrophy (ANOVA).

${ }^{a}$ Accession numbers of the microarray set 
Fig. 6 Networks of functionally related genes with a sex-specific regulation. Ingenuity Pathways Analysis was used to create networks derived from the two sets of genes with a relative upregulation in females (a) or in males (b; $p<0.05$, ANOVA statistics). For each set, the network with the highest score is shown. Different combinations of the directions of deregulation in the comparison of TAC and sham for males and females could lead to a sex-specific expression pattern as indicated by the color code. Linking nodes not contained in the two sets of genes are colored gray. A number of genes in the female network (a) are related to energy metabolism (marked with green stars). They are grouped around PGC-1 (PPARGC1A, peroxisome proliferative activated receptor, gamma, coactivator 1 , alpha). In contrasting, many of the genes in the male network are related to changes of extracellular matrix (highlighted with yellow stars) or biosynthesis (pink stars). The complete list of genes of the networks can be seen in Table e7 (Electronic supplementary material)

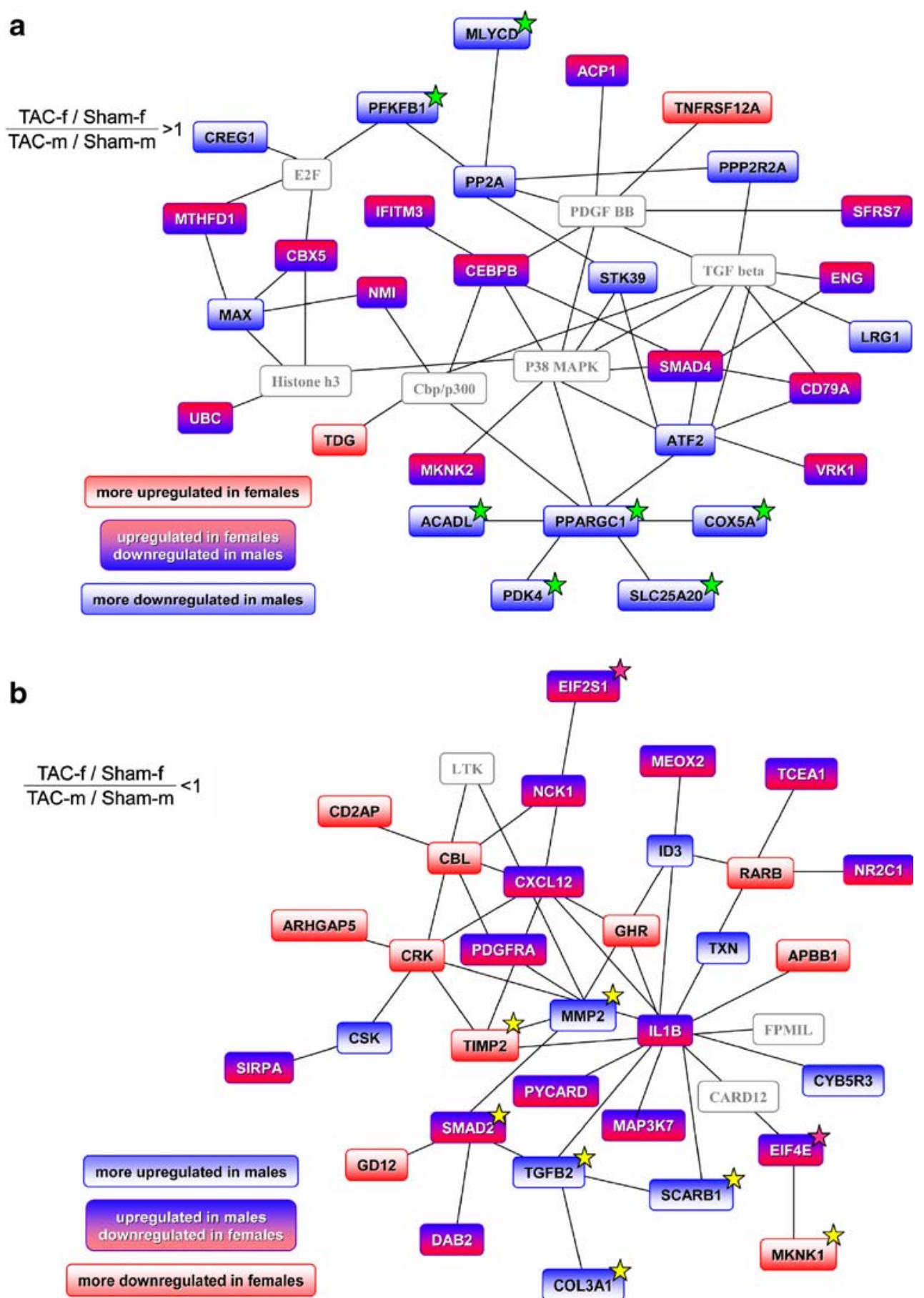

orientation showed no E2 inducibility in the presence of ER $\alpha$. These data indicate that the first $600 \mathrm{bp}$ of the PGC- $1 \alpha$ promoter are sufficient to obtain the E2-ER $\alpha$-mediated effect.

\section{Discussion}

We found that many genes were differentially regulated in male and female mice before sex-specific morphological or functional changes occurred. We consigned these genes to networks that feature the predominant early response in the male and female hearts to PO. Less downregulation of genes encoding mitochondrial function and fatty acid oxidation characterized the network of the female hearts and relative upregulation of matrix remodeling and ribosomal genes the network of the male hearts. The data support the concept that better adaptation of energy metabolism in the female hearts, and greater induction of matrix turnover and ribosomal protein synthesis in the male hearts, are important mediators of sex-dependent remodeling during early stages of PO. These 


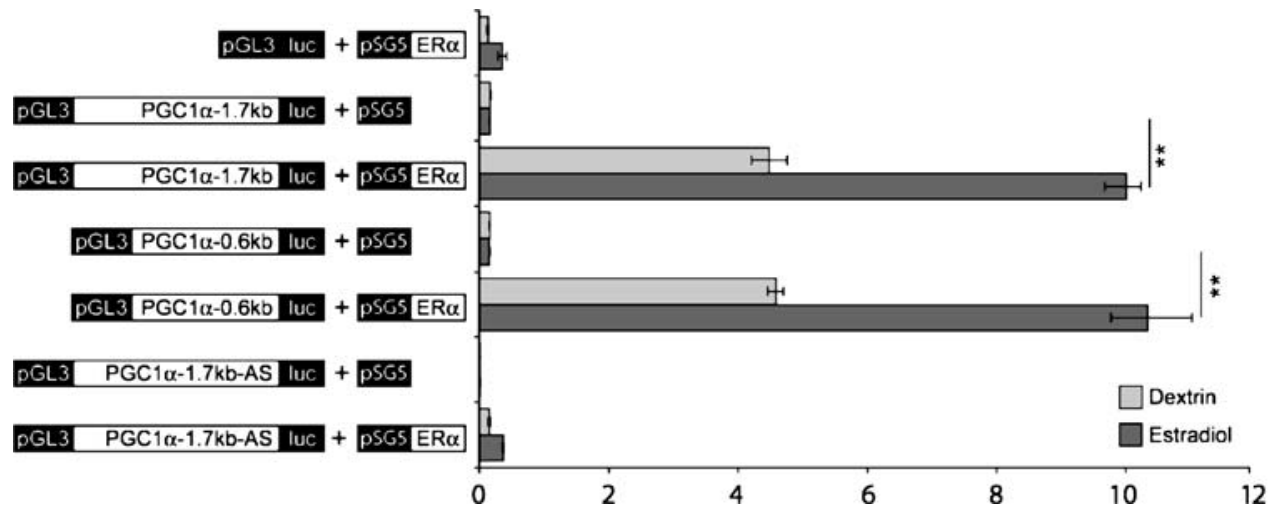

Fig. 7 Effect of E2 and ER $\alpha$ on the transcriptional activity of the PGC- $1 \alpha$ promoter. PGC- $1 \alpha$ luciferase reporter constructs were cotransfected with or without ER $\alpha$-pSG5 plasmid, along with Renilla luciferase reporter construct into AC16 cells, and the cells were then treated with estrogen $\left(\mathrm{E} 2,10^{-8} \mathrm{M}\right)$ or left untreated. After $24 \mathrm{~h}$, the

processes may represent the molecular basis for the sexspecific functional changes observed at late stages of $\mathrm{PO}$ and LVH.

In transgenic LVH models, HF and death occur earlier in male than in female animals [14-16, 31]. TAC-induced PO represents a global stimulus for LVH that is initially concentric and leads at later stages to dilatation and HF. Rats and mice exhibit sex differences in the LVH response to PO and the transition to HF, which are accompanied by changes in gene expression [17-20,32]. However, sex differences in gene expression that occur after the manifestation of sex differences in cardiac function may represent a response to differences in hemodynamic load rather than its cause. We therefore searched to detect the early sex differences in gene expression following TAC that may determine the sex differences in cardiac function at later stages. We euthanized mice 2 weeks after TAC to measure gene expression at a time point when hypertrophy was already present, yet without sex differences. LVH-related sex differences developed only at later stages after TAC in our model, similar to other reports $[17-19,33]$. To obtain evidence that our microarray analysis had sufficient sensitivity and specificity to detect biologically relevant gene expression changes, we analyzed gene expression profiles of classical markers of hypertrophy and sex chromosome-linked genes. Indeed, we observed the upregulation of a comprehensive hypertrophy marker set induced by TAC as described earlier, as well as $\mathrm{Y}$ chromosomal genes in males [21, 22, 34].

A significant overrepresentation of male-upregulated genes was found in the gene ontology categories of biosynthesis and cell organization, which agrees with published data analyzing the effects of TAC on gene expression and showing strong activation of matrix remodeling [21, 22, 34]. A group of ribosomal genes linked to protein biosynthesis also showed relative upregulation in male hearts, which may be explained luciferase activity was measured and normalized to the Renilla luciferase activity in each experiment. The graph shows the results of three independent experiments performed in triplicates, expressed as mean values \pm SEM. ${ }^{* *} p<0.01$ vs. co-transfection without ER $\alpha$ pSG5 plasmid

by the greater need of protein synthesis for increased matrix turnover. Accordingly, the most prominent male network assembles matrix regulating genes, namely collagens, matrix metalloproteinases (MMP), and tissue inhibitors of MMPs (TIMPs). An increase of MMP14 after PO in males was also reported earlier in a similar setting [19]. Our data correspond well to the analyses of PO-induced gene expression in a similar TAC model by Mirotsou et al. [22]. First, a large number of hypertrophy-related genes that they found to be regulated were also detected in our study, including ANP, collagens, and CTGF. Second, genes with functions related to cell growth, morphology, differentiation, and ECM activity were directly correlated with $\mathrm{LVH}$, which is also in agreement with our study showing upregulation of matrix-related and ribosomal genes, predominantly and most pronounced in males [22]. Based on our findings, male TAC mice have a higher collagen turnover than females, which may lead to an altered collagen composition with a different amount of cross linking and collagen subtypes in both sexes. Indeed, the modulation of extracellular matrix synthesis has been linked to sex differences in cardiac stiffness underlying sex differences in diastolic function in clinical syndromes $[6,17,20]$.

Female hearts were characterized by the relatively better maintenance of metabolic capacity. An unbiased analysis placed the PPAR $\gamma$-coactivator $1 \alpha$ (PGC-1) in a central position in the most relevant female network [35]. PGC-1 stimulates mitochondrial biogenesis and fatty acid oxidation and reduces glucose oxidation in the myocardium [36, 37]. Accordingly, we found less downregulation of PGC-1controlled genes involved in mitochondrial fatty acid metabolism and the respiratory chain in females. Examples included acyl-CoA dehydrogenase, carnitine-palmitoyl transferase, cytochrome oxidase, and pyruvate dehydrogenase kinase 4 , which downregulates glucose metabolism. Mirotsou et al. also found an inverse correlation of energy metabolism- 
related genes and mitochondrial function with left ventricular hypertrophy after TAC in a cohort of females and males, which was confirmed by our data with the additional finding of a stronger downregulation in males than in females [22]. Recent work by Wagner et al. in male hearts also showed that mitochondrial energy metabolism is decreased after TAC [34]. O'Lone et al. demonstrated that a large number of energy metabolism-related and mitochondrial function-related genes are controlled by estrogen via $\mathrm{ER} \alpha$ or $\mathrm{ER} \beta$ in the vasculature [24]. This finding corresponds well to the group of genes that were upregulated in our female network, namely genes associated with energy metabolism, mitochondrial genes, and genes involved in electron transport and fatty acid oxidation. Interestingly, Haddad et al. found in a gene expression profiling on idiopathic dilated cardiomyopathy in humans that metabolic genes are mainly deregulated in females and genes related to muscular contraction and extracellular matrix in males [38]. Altogether these studies indicate that our findings are not restricted to our model, but represent general mechanisms in the sex-specific adaptation processes of the heart.

PGC-1 transcription is tightly controlled by the family of myocyte enhancer factors (MEF) including MEF2c [14]. Mef2c is a transcription factor regulated by the family II of histone deacetylases (HDAC) and signaling pathways that utilize calcineurin, calmodulin-dependent protein kinase, and MAPKs. MEF2c overexpression induces the fetal gene program and leads to dilated cardiomyopathy [39]. Relative downregulation of MEF2c in our female mouse hearts may limit the activation of the hypertrophic gene program following stress and thereby exert protective effects. However, MEF2c also stimulates mitochondrial biogenesis and function via upregulation of PGC-1 [14]. Reduction of MEF2c activity would therefore be expected to lead to a relative downregulation of PGC-1 and of mitochondrial biosynthesis. Indeed, in a mouse model with inducible repression of MEF by permanent HDAC 5 activation, all male mice died within 5-10 days after transgene induction, with highly impaired mitochondrial structure and function [14]. However, female mice survived about 30 days with signs of a different pathogenic process but with normal mitochondrial function. Based on our data, showing a significant downregulation of MEF2c but normal PGC-1 expression in hypertrophic female hearts, we suggest that female mice can maintain PGC-1 independent of MEF2c. This capability could allow them to sustain mitochondrial function in hypertrophic states. This is supported by our finding of the transcriptional control of the PGC-1 gene by E2 and with the already documented upregulation of PGC-1 by E2 in vivo, which has protective effects and restores mitochondrial ATP production, thus preventing cardiac depression after trauma-hemorrhage [40, 41]. The unaltered MEF2c gene expression level in banded males and the decrease in banded females may thus be involved in the observed morphological and functional sex-dependent changes at later stages of hypertrophy. The female heart may maintain an increased cardiac work load under stress by improved energy supply due to relatively stronger activation of mitochondrial function and fatty acid oxidation at a lower degree of activation of a hypertrophic gene profile (Fig. 8). The upregulation of PGC-1 by estrogen can explain the more favorable response of the female myocardium to hemodynamic load and provide a molecular basis for myocardial protection in female hearts. Endogenous upregulation of PGC-1 by estrogen is likely to be more favorable than forced cardiac overexpression of PGC-1 leading to cardiomyopathy and heart failure [42].

\section{Limitations of the study}

Our findings on sex differences in hypertrophy-related gene expression in mice are consistent with the concepts discussed in previous reports [17, 19]. Nevertheless, larger assay numbers are needed to analyze age dependency and mouse strain specificity. To describe the pathways involved and to prove their mediating role, more experimental approaches are required that alter the function of one or more of the genes identified.

Species differences between humans and mice have to be analyzed in further studies as well as the effect of hormonal status of the females. We found that employing synchronized females did not reduce inter-animal variability in gene expression [43]. Finally, to work out the whole complexity of sex-specific responses in hypertrophy, systems biology, proteomic, and metabolomic approaches must be developed to complement the expression studies.

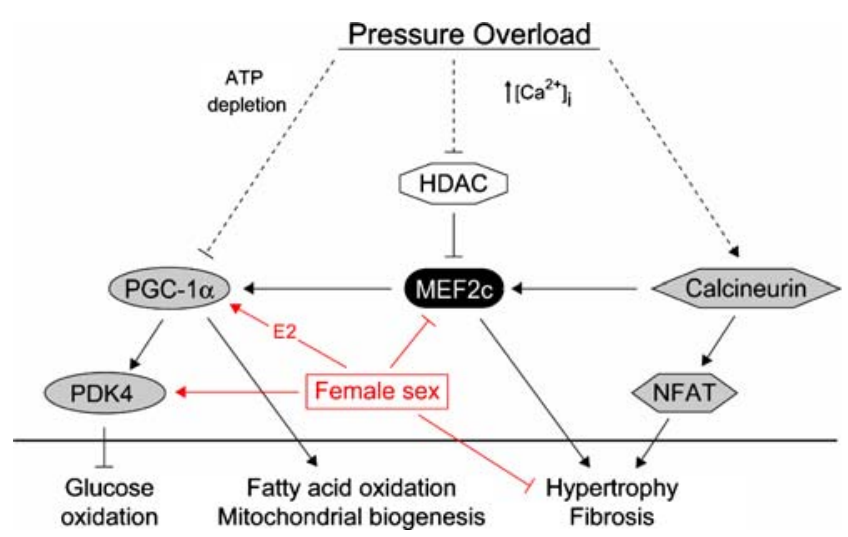

Fig. 8 Hypothesis of sex differences in the hypertrophic response to pressure overload. In female mice, a relative higher expression of PGC-1 and PDK4 was observed 2 weeks after TAC. This may lead to a sustained level of fatty acid oxidation and inhibit an increase of glucose oxidation, indicating an attenuation of the metabolic switch in the female myocardium after TAC. The relative downregulation of $\mathrm{MEF} 2 \mathrm{c}$ may reduce the hypertrophic gene program and fibrosis 


\section{Conclusion}

In the early response to pressure overload, a more efficient metabolic regulation in female hearts, and greater induction of matrix remodeling in male hearts, may set the stage for the relative protection of the female hearts. Translation of these findings to human PO-induced hypertrophy and HF could benefit both women and men.

Acknowledgments We gratefully acknowledge the critical comments of Noel Bairey Merz, Medical Director and Endowed Chair, Women's Health Program, Cedars-Sinai Medical Center and Friedrich Luft, Director of the Experimental and Clinical Research Center in Berlin-Buch. We thank Pierre Chambon (Institute of Genetics, Molecular and Cellular Biology, CNRS/INSERM, College de France, Illkirch Cedex, France) for his of ER $\alpha$-pSG5 plasmid (HEGO vector). We thank Stefanie Roehner for excellent secretarial work and Jenny Thomas for excellent technical assistance. The EU FP6 grant LSHMCT-2005-018833, EUGeneHeart, the Deutsche Forschungsgemeinschaft Graduate Studies Grant 754 on myocardial hypertrophy, DFG Re 662/6-1, and Grohe 729/12-1 supported this work. We thank Joerg Isensee for helping with the graphical illustrations. Parts of the doctoral thesis of Juliane Jaekel have been incorporated into this article.

\section{References}

1. Carroll JD, Carroll EP, Feldman T, Ward DM, Lang RM, McGaughey D, Karp RB (1992) Sex-associated differences in left ventricular function in aortic stenosis of the elderly. Circulation 86:1099-1107

2. Aurigemma GP, Gaasch WH (1995) Gender differences in older patients with pressure-overload hypertrophy of the left ventricle. Cardiology 86:310-317

3. Aurigemma GP, Silver KH, McLaughlin M, Mauser J, Gaasch WH (1994) Impact of chamber geometry and gender on left ventricular systolic function in patients $>60$ years of age with aortic stenosis. Am J Cardiol 74:794-798

4. Villari B, Campbell SE, Schneider J, Vassalli G, Chiariello M, Hess OM (1995) Sex-dependent differences in left ventricular function and structure in chronic pressure overload. Eur Heart J 16:1410-1419

5. Hogg K, Swedberg K, McMurray J (2004) Heart failure with preserved left ventricular systolic function; epidemiology, clinical characteristics, and prognosis. J Am Coll Cardiol 43:317-327

6. Regitz-Zagrosek V, Brokat S, Tschope C (2007) Role of gender in heart failure with normal left ventricular ejection fraction. Prog Cardiovasc Dis 49:241-251

7. Cleland JG, Swedberg K, Follath F, Komajda M, Cohen-Solal A, Aguilar JC, Dietz R, Gavazzi A, Hobbs R, Korewicki J, Madeira HC, Moiseyev VS, Preda I, van Gilst WH, Widimsky J, Freemantle N, Eastaugh J, Mason J (2003) The EuroHeart Failure survey programme - a survey on the quality of care among patients with heart failure in Europe. Part 1: patient characteristics and diagnosis. Eur Heart J 24:442-463

8. Simon T, Mary-Krause M, Funck-Brentano C, Jaillon P (2001) Sex differences in the prognosis of congestive heart failure: results from the Cardiac Insufficiency Bisoprolol Study (CIBIS II). Circulation 103:375-380

9. O’Meara E, Clayton T, McEntegart MB, McMurray JJV, Pina IL, Granger CB, Ostergren J, Michelson EL, Solomon SD, Pocock S, Yusuf S, Swedberg K, Pfeffer MA (2007) Sex differences in clinical characteristics and prognosis in a broad spectrum of patients with heart failure: results of the candesartan in heart failure: assessment of reduction in mortality and morbidity (CHARM) program. Circulation 115:3111-3120

10. Mendelsohn ME, Karas RH (2005) Molecular and cellular basis of cardiovascular gender differences. Science 308:1583-1587

11. Modena MG, Muia N Jr, Aveta P, Molinari R, Rossi R (1999) Effects of transdermal 17beta-estradiol on left ventricular anatomy and performance in hypertensive women. Hypertension 34:1041-1046

12. Regitz-Zagrosek V (2006) Therapeutic implications of the genderspecific aspects of cardiovascular disease. Nat Rev Drug Discov $5: 425-438$

13. van Eickels M, Grohe C, Cleutjens JP, Janssen BJ, Wellens HJ, Doevendans PA (2001) 17Beta-estradiol attenuates the development of pressure-overload hypertrophy. Circulation 104:1419-1423

14. Czubryt MP, McAnally J, Fishman GI, Olson EN (2003) Regulation of peroxisome proliferator-activated receptor gamma coactivator 1 alpha (PGC-1 alpha) and mitochondrial function by MEF2 and HDAC5. Proc Natl Acad Sci U S A 100:1711-1716

15. Du XJ (2004) Gender modulates cardiac phenotype development in genetically modified mice. Cardiovasc Res 63:510-519

16. Leinwand LA (2003) Sex is a potent modifier of the cardiovascular system. J Clin Invest 112:302-307

17. Weinberg EO, Mirotsou M, Gannon J, Dzau VJ, Lee RT, Pratt RE (2003) Sex dependence and temporal dependence of the left ventricular genomic response to pressure overload. Physiol Genomics $12: 113-127$

18. Weinberg EO, Thienelt CD, Katz SE, Bartunek J, Tajima M, Rohrbach S, Douglas PS, Lorell BH (1999) Gender differences in molecular remodeling in pressure overload hypertrophy. J Am Coll Cardiol 34:264-273

19. Skavdahl M, Steenbergen C, Clark J, Myers P, Demianenko T, Mao L, Rockman HA, Korach KS, Murphy E (2005) Estrogen receptor-beta mediates male-female differences in the development of pressure overload hypertrophy. Am J Physiol Heart Circ Physiol 288:H469H476

20. Douglas PS, Katz SE, Weinberg EO, Chen MH, Bishop SP, Lorell BH (1998) Hypertrophic remodeling: gender differences in the early response to left ventricular pressure overload. J Am Coll Cardiol 32:1118-1125

21. Colston JT, Boylston WH, Feldman MD, Jenkinson CP, de la Rosa SD, Barton A, Trevino RJ, Freeman GL, Chandrasekar B (2007) Interleukin-18 knockout mice display maladaptive cardiac hypertrophy in response to pressure overload. Biochem Biophys Res Commun 354:552-558

22. Mirotsou M, Dzau VJ, Pratt RE, Weinberg EO (2006) Physiological genomics of cardiac disease: quantitative relationships between gene expression and left ventricular hypertrophy. Physiol Genomics 27:86-94

23. Zhao M, Chow A, Powers J, Fajardo G, Bernstein D (2004) Microarray analysis of gene expression after transverse aortic constriction in mice. Physiol Genomics 19:93-105

24. O’Lone R, Knorr K, Jaffe IZ, Schaffer ME, Martini PG, Karas RH, Bienkowska J, Mendelsohn ME, Hansen U (2007) Estrogen receptors alpha and beta mediate distinct pathways of vascular gene expression, including genes involved in mitochondrial electron transport and generation of reactive oxygen species. Mol Endocrinol 21:1281-1296

25. Dennis G Jr., Sherman BT, Hosack DA, Yang J, Gao W, Lane HC, Lempicki RA (2003) DAVID: Database for Annotation, Visualization, and Integrated Discovery. Genome Biol 4:P3

26. Hosack DA, Dennis G Jr., Sherman BT, Lane HC, Lempicki RA (2003) Identifying biological themes within lists of genes with EASE. Genome Biol 4:R70

27. Rozen S, Skaletsky H (2000) Primer3 on the WWW for general users and for biologist programmers. Methods Mol Biol 132:365386 
28. Tusher VG, Tibshirani R, Chu G (2001) Significance analysis of microarrays applied to the ionizing radiation response. Proc Natl Acad Sci U S A 98:5116-5121

29. Davidson MM, Nesti C, Palenzuela L, Walker WF, Hernandez E, Protas L, Hirano M, Isaac ND (2005) Novel cell lines derived from adult human ventricular cardiomyocytes. J Mol Cell Cardiol 39:133-147

30. Rinn JL, Rozowsky JS, Laurenzi IJ, Petersen PH, Zou K, Zhong W, Gerstein M, Snyder M (2004) Major molecular differences between mammalian sexes are involved in drug metabolism and renal function. Dev Cell 6:791-800

31. Djouadi F, Weinheimer CJ, Saffitz JE, Pitchford C, Bastin J, Gonzalez FJ, Kelly DP (1998) A gender-related defect in lipid metabolism and glucose homeostasis in peroxisome proliferatoractivated receptor alpha-deficient mice. J Clin Invest 102:1083-1091

32. Tamura T, Said S, Gerdes AM (1999) Gender-related differences in myocyte remodeling in progression to heart failure. Hypertension 33:676-680

33. Loyer X, Oliviero P, Damy T, Robidel E, Marotte F, Heymes C, Samuel JL (2007) Effects of sex differences on constitutive nitric oxide synthase expression and activity in response to pressure overload in rats. Am J Physiol Heart Circ Physiol 293:H2650-H2658

34. Wagner RA, Tabibiazar R, Powers J, Bernstein D, Quertermous T (2004) Genome-wide expression profiling of a cardiac pressure overload model identifies major metabolic and signaling pathway responses. J Mol Cell Cardiol 37:1159-1170

35. Arany Z, He H, Lin J, Hoyer K, Handschin C, Toka O, Ahmad F, Matsui T, Chin S, Wu PH, Rybkin II, Shelton JM, Manieri M, Cinti S, Schoen FJ, Bassel-Duby R, Rosenzweig A, Ingwall JS, Spiegelman BM (2005) Transcriptional coactivator PGC-1 alpha controls the energy state and contractile function of cardiac muscle. Cell Metab 1:259-271
36. Barger PM, Browning AC, Garner AN, Kelly DP (2001) p38 mitogen-activated protein kinase activates peroxisome proliferator-activated receptor alpha: a potential role in the cardiac metabolic stress response. J Biol Chem 276:44495-44501

37. Lehman JJ, Kelly DP (2002) Transcriptional activation of energy metabolic switches in the developing and hypertrophied heart. Clin Exp Pharmacol Physiol 29:339-345

38. Haddad GE, Saunders LJ, Crosby SD, Carles M, del Monte F, King K, Bristow MR, Spinale FG, Macgillivray TE, Semigran MJ, Dec GW, Williams SA, Hajjar RJ, Gwathmey JK (2008) Human cardiacspecific cDNA array for idiopathic dilated cardiomyopathy: sexrelated differences. Physiol Genomics 33:267-277

39. Xu J, Gong NL, Bodi I, Aronow BJ, Backx PH, Molkentin JD (2006) Myocyte enhancer factors 2A and 2C induce dilated cardiomyopathy in transgenic mice. J Biol Chem 281:9152-9162

40. Hsieh YC, Yang S, Choudhry MA, Yu HP, Bland KI, Schwacha MG, Chaudry IH (2006) Flutamide restores cardiac function after trauma-hemorrhage via an estrogen-dependent pathway through upregulation of PGC-1. Am J Physiol Heart Circ Physiol 290: H416-H423

41. Hsieh YC, Yang S, Choudhry MA, Yu HP, Rue LW 3rd, Bland KI, Chaudry IH (2005) PGC-1 upregulation via estrogen receptors: a common mechanism of salutary effects of estrogen and flutamide on heart function after trauma-hemorrhage. Am J Physiol Heart Circ Physiol 289:H2665-H2672

42. Lehman JJ, Barger PM, Kovacs A, Saffitz JE, Medeiros DM, Kelly DP (2000) Peroxisome proliferator-activated receptor gamma coactivator-1 promotes cardiac mitochondrial biogenesis. J Clin Invest 106:847-856

43. Isensee J, Witt H, Pregla R, Hetzer R, Regitz-Zagrosek V, Ruiz Noppinger P (2008) Sexually dimorphic gene expression in the heart of mice and men. J Mol Med 86:61-74 